\title{
Risk Factor in the Rehabilitation Process of Forensic Patients
}

\author{
Phd. Eglantina Dervishi \\ Head of psychology Department, Albanian University \\ egladervishi@gmail.com
}

\section{Doi:10.5901/ajis.2014.v3n6p17}

Abstract

This study investigates the risk factors on rehabilitation process of forensic patients in the psychiatric department in Prison General Hospital in Tirana, Albania. A sample of 89 forensic patient was include on this study. Who-Das questionnaire was used to elicit information from the nursery staff. The result showed that a significant relationship exist between the dysfunctional behavior of each forensic patient, the level of severity of their disorder and the rehabilitation process.

\section{Introduction}

Nowadays, the psychiatric assistance is facing a lot of challanges in its attempts to change its the intensive intervention line at the persons with severe mental health problems Zhang et al., (2011). The realisation of the rehabilitation process is a competence of psychiatric institutions one of which is the Prison General Hospital, Tirana. This hospital is in charge of treating forensic patients who do not deal only with medical physical problems but also with mental problems. These persons with mental health issues are sentenced with the "obligated medication" detention and are subject of the rehabilitation treatment of the multidisiplinary team of the institution. Moreoften the staff of the institution face different problems in the treating process due to the risk factors which interfere with the in the personal building capacity Gruneir et al., (2012) and in the improving of their life quality Honkonen et al.,(2008). According to Corrigan et al, (1998) patients who have a reduced number of acute symptomatology have a higher probability to succeed in a successful way the rehabilitation process, while the opposite happens when the patients who display a large range of symptomatology. For them it is much harder to positively respond to a good rehabilitation plan.

\section{The Problem}

The motivation to develop this study came from the need to contribute in the research area which aims to determine the risking factors which inhibit people with mental health problem to develop their potential Steel et al., (2011), and that slow the rehabilitation plan implemented by the multidisciplinary team of the institution Vigod et al., (2013). The slow down of the rehabilitation plan is strongly connected with the problematic behavior displayed by patients in the institution. Some of the risk factors like patient behavior, the contact with the outside world, the occupation of patient in the department, specific resources of the patient and specific risk factors play important roles in understanding the dynamics that orients or not the patients toward a fast rehabilitation. Researches believe that there is a connection between the gravity of symptoms and the success of the rehabilitation process for the psychiatric patient Jones \& Crossley, (2008). Therefore, this is also the goal of our study: to discover if there is a possibility that the risk factors related to forensic patients behavior would interfere with their rehabilitation, increasing so on even the difficulty of working staff.

\section{Purpose}

The goal of this study is to explore forensic patients behavior in the institution focusing on the elements which show dysfunction in social behavior and roles and the aggravated symptomatology that results from mental sickness. The results of this study will help with the elaboration of an individual plan which takes into account the gravity of sickness and the risk factors while creating a successful rehabilitation program. 


\section{Hypotheses}

There is a significant relationship between patient's behavior in institution and his mental health conditions. There is a significant relationship between social support and the rehabilitation process of forensic patients.

\section{Methodology}

An exploring study is designed with the main goal to identify the gravity of psychiatric symptoms of forensic patients and its connection to the rehabilitation work of the staff.

Population and sample: The population of the study is a group of 89 patients resident in psychiatric department in the General Hospital of Prision in Tirana. From this population, there were 79 males and 10 females. This is a representative sample since we included all the patients in the Hospital Center of Prisons which are forensic patients treated for different mental health issues. This Prison covers all the Albanian territory.

Instrument: The instrument used is called Who- Das. It is a 32 questions scale on Liker style. This instrument explore patient's behavior, specific risk factors, specific resources of the patients, his occupation in the sector, his contacts with the outside world and the personal file of patient.

\section{Results}

Who-Das is an instrument filled by the nurses and psychologists of the sector concerning patient's data in different areas which investigate the behavior and patients' functioning in the sector. The inner validity expressed in terms of alpha Cronbach (a .701) resulted to be significant according to the authors of the instrument Ardoin et al.,(1991), cit.in Ruggeri et al.,(2000). In all the sub scales, the data show that patients' behavior is affected in different areas like: his behavior in the sector $(M=0.59, S D=0.35)$. These values show a malfunctioning in different area of self care like: personal hygiene, self dressing, self feeding etc. Factors of specific risks show these results $(M=0.77, S D=0.43)$ as related to a slowdown motor skills. Through patient's opinion, we discovered that the environment patient grow up in, influenced his attitude. This context is described like: being part of a disadvantaged group, appropriate living conditions, a series of economical and social problems, poverty, family context disorganized, patients health or other family member issues, ( $\mathrm{M}=0.67$, $\mathrm{SD}=0.23$, ) the occupation of patients in the hospital is inexistent $(\mathrm{M}=0.91, \mathrm{SD}=0.34)$. For all the sub scales of the questionnaire, we found out an aggravated symptomatology in all patients ( $F=15.3 \mathrm{df}=8$ and $792, p<0.01)$. Also from the items related to the way to find pleasure ( $M=0.70, v 0.42)$, violent and risky behavior $(M=0.85, v 0.72)$, social withdraw $(\mathrm{M}=0.53, v 0.39)$ show a difference that define the compromised area in client behavior. There are also made some analyses of multivariate regression with the step wise methods in order to identify the factors that influence patient's behavior in hospital like: gender, civil status, the committed act, diagnose and the time stayed to the hospital. From the analyses is concluded that one predictor factor that do not emerge a violent behavior is being a widow which is different from being single, married or divorced, $\beta=-3.40 \mathrm{df}=23 \mathrm{p}=0.05$, while related to familiar context, it is shown that males $\beta=-$ $3.70 \mathrm{df}=22 \mathrm{p}=0.03$ have more difficulties in keeping contacts and supporting the family. The motor slowdown is more evident in forensic patients with single as a civil status $\beta=-2.11$, Wald $=4.9, p=0.02$, and with a schizo- affective disorder $\beta=-3.47$, Wald $=4.8, p=0.02$.

Regression analyses showed that only the widow patients did not have the tendency to display violent and risky behavior. Single forensic patients which represent also our wider target in the study, age 35-45 display a non positive attitude toward the family, determined also from a lack of orientation figure in the family. For the married patients, family atmosphere is more positive and satisfactory. Single patients with schizophrenic diagnose, aging $35-45$ years old display a wide range of disfunction's related to the time they need to dress, their motor skills, in eating showing more compromised behavior and functioning. These patients are less socially involved and make social contacts only if needed or encouraged by the staff.

Females with a psycho affective diagnose are more involved in compulsive behavior concerning hygiene while men with the same diagnose neglected it more. Male patients with a single civil status are the more violent and aggressive. There is no difference concerning the age or the diagnoses. In the end, we can accept the fact that the high level of problematic behavior in forensic patients will deeply affect their personal empowering. 


\section{Discussion}

Rehabilitation is a process achieved through therapeutic assistance offered by psychiatric staff of the hospital. Mitchell (2003) defines rehabilitation as a working tool of mental health professionals. They apply many rehabilitating activities to help mental health patients to win and apply new abilities, to ask support and to use all their means in order to live a fulfilled life outside the institutions. Rehabilitation process is compromised by many risk factors.

The data of this study needs further and deeper researches about the reliability and validity. Even though, this study will be one of the first which gives a clear overview on the risky factors which affect the maximizing of forensic patients potential, and explain their functioning and social difficulties. Different studies have assumed Corrigan et al, (1998) that persons with "reduced symptomatology" and normal overall functioning appear more empowered. Who - Das sub scale for hospitalized patients, showed an accepted behavior of patients concerning the: speed to move, takj, social relationship, taking care and their behavior during meals. An important element that needs to be discussed is the high presence of threatening behavior against others. Some of the risk factors that influence this aggressive behavior are: being part of an disadvantaged group, serious economical difficulties and poverty, the presence of physic and neurologist deficits of clients, a disorganized family environment. According to nurses' opinion, one of the hardest thing is the motivation of patients toward rewarding activities. Even though some of them show interests in different area like reading, writing, drawing and games it does not last for long time and the skills do not improve.

Another condition which directly affect the rehabilitation process is the lack of occupational activities in the sector. This is a wide problem mostly in acute psychiatric patients Sundram (1987). In fact, most of the patients pass most of their time in their cells, closed. Any form of occupational therapy is very hard to be implemented in prison hospital due to the high risk measures.It is forbidden to keep tools or things that can constitute a risk for patients' life. Most of their activities is limited to one hour air a day, in a surrounded area with concrete. Lack of structure and access to rehabilitation process do not help the maximizing of patient's potential Smith \& Roberts (1996). Another risk factor which interfere with the rehabilitation process is patients' isolation Jones \& Crossley (2008). Contacts with the outside world seem to be compromised. Family visit are applied once a moth and there is never applied the home visit. Family presence is a strong point to support patients and are seen as a sign of his personal empowerment Hutcheson et al., (2010). In our case, this relationship is not used as an indicator of personal empowering of patients. For a big part of patients $(82,4 \%)$ families live far from the institutions making it impossible for them to travel, attend meetings or to provide their beloved in the institutions with goods (food and clothes).

\section{Conclusion}

At the end, we confirm that the risky factors in rehabilitation process of psychiatric patients are the biggest challenge of the research in our time, even though we keep up the optimism. We believe that the risk factors are a clinical risk and a barrier in the rehabilitation process. Sickness gravity, the isolation due to measure of arrest "obligated medications" directly affect the improvement process of forensic patients.A successful intervention program at these patients should take into consideration the risk factors which often interfere with the rehabilitation plan applied by the institution and postpone the integration of patients into the community.Every forensic patients have the right to live with dignity and to be treated as such from the family, the staff and the society.Further researches on risk factors might orient us into the developing of better standards for clinical care toward this category.

\section{References}

Corrigan P.W., Faber D., Rashid F., Leary M. (1998) The construct validity of empowerment among consumers of mental health services. (electronic version)., Schizofrenia Research; 38 (1), 77-84

Gruneir A, Bronskill S, Bell C, et al. (2012). Recent health care transitions and emergency department use by chronic long term care residents: a population-based cohort study. J Am Med Dir Assoc.; 13(3):202-206

Honkonen H., Mattila Ak., Lehtinen K., et al. (2008) Motrality of Finish acute psychiatric hospital patients. Soc Psychiatry Psychiatr Epidemiol 43; 660-666.

Hutcheson C., Ferguson H., Nish G., Gill L. (2010)Promoting Mental Wellbeing throught Activity in a Mental Health Hospital. British Journal of Occupational Therapy. Volume 73, (3), 121- 34

Jones A \& Crossley D. (2008) "In the minde of another" shame and acute psychiatric inpatient care: an exploratory study. A raport of fase one:service users. Journal of psychiatric and Mental Health Nursing; 15 (9), 749-757.

Jones A, Crossley D. (2008) "In the mind of another" shame and acute psychiatric inpatient care; an exploratory study. A report of phase one: service users. Journal of Psychiatric and Mental Health Nursing; 15 ( 9), 749-57 
Mitchell S. (2003) Rehabilitation and recovery. Auseinet,19, November; 13-15.

Smith J, Gross C, Roberts J. (1996)The evalution of the therapeutic environment for patients with long-term mental illness as measured by the Ward Atmosphere Scale. Journal of Mental Health 1996; 5 (4), 349-60

Steel Z, Momartin S, Silove D, et al., (2011). Two year psychosocial and mental health outcomes for refugees subjected to restrictive or supportive immigration policies. Soc Sei Med. ;72(7): 1149-1156

Sundram C.Patient idleness in public mental hospital. Psychiatric Quarterly 1987; 58 (4), 243-54

Vigod SN, Kurdyak PA, Dennis CL, et al. (2013). Transitional interventions to reduce early psychiatric readmissions in adults: systematic review. Br J Psychiatry;202(3): 187-194

Zhang J, Harvey C, Andrew C. (2011). Factors associated with length of stay and the risk of readmission in an acute psychiatric inpatient facility: a retrospective study. Aust N Z J Psychiatry. ;45(7):578-585 